\title{
ENSAYOS
}




\section{ESCUELA, MARGINALIDAD Y CONTEXTOS SOCIALES EN COLOMBIA}

\section{Rodrigo Parra S.* Leonor Zubieta*}

El tema de este artículo constituye la síntesis de los hallazgos más significativos de dos experiencias realizadas en Colombia con el propósito de estudiar, desde una perspectiva sociológica, la relación educación y marginalidad. La primera de ellas fue efectuada en una comunidad marginal urbana ${ }^{1}$ y la segunda en una campesina ${ }^{2}$. Una y otra se ubican en el marco de las consideraciones teóricas y metodológicas siguientes:

1. Entre las múltiples caras que puede tener una realidad como la configurada por las relaciones educación y sociedad, la del proceso de socialización que se supone realiza la institución educativa es tan aceptada como desconocida. Se ignora, por ejemplo, a) cuál es la naturaleza y cuál el significado de la socialización lograda, o por lo menos intentada, por la escuela en diversos contextos sociales, b) qué implicaciones tienen para la imagen del maestro y de la escuela sus roles de agentes socializadores, c) qué tipo de factores inciden y de qué manera en los distintos ámbitos sociales en los propósitos y resultados de la socialización vía institución educativa.

Los estudios mencionados permiten adelantar una primera reflexión sobre el tema para el caso concreto de comunidades marginales, como se verá más adelante. Los resultados de otros estudios posibilitan aventurar, por lo menos a manera de hipótesis, algunos planteamientos sobre la situación educativa en contextos sociales diferentes.

2. La metodología empleada en cada uno de los trabajos constituye una aproximación al enfoque cualitativo de la etnografía. Ninguno de ellos desconoce el aporte que ha significado para el conocimiento del sistema educativo colombiano la información proveniente de los estudios cuantitativos como aquellos dedicados al diagnóstico de su estado y eficiencia, pero cuando se trata de conocer las funciones reales que cumple la escuela, de precisar los significados sociales de conceptos como educación, rol docente, imagen del maestro, vida escolar, relaciones escuela-comunidad, etc., se necesita también una postura metodológica diferente que, como la de la etnografía, permita la aprehensión de la realidad que se estudia desde su cotidianidad, su multiplicidad y la subjetividad de sus actores.

\section{Socialización y realidad subjetiva}

El proceso mediante el cual el nuevo individuo aparecido en la sociedad llega a ser realmente un ser social, a "aprehender el mundo como realidad significativa y social" y a encontrar un lugar para él en la estructura se conoce como el proceso de socialización.

\footnotetext{
* Sociólogo U. Nacional, M.A. en Sociología. U. de Wisconsin; Ph D En Sociología, U. de Wisconsin, Investigador Centro de Investigaciones. Universidad Pedagógica Nacional.

Trabajadora Social. Universidad Externado de Colombia. Estudios de Postgrado en Investigación Socioeducativa Universidad Pedagógica Nacional. Investigadora del Centro de Investigaciones de la Universidad Pedagógica (CIUP).

1 Juan Carlos Tedesco y Rodrigo Parra, Marginalidad Urbana y Educación Formal, Buenos Aires, Proyecto Desarrollo y Educación en América Latina y el Caribe. UNESCO-CEPAL-PNUD. 1980.

${ }_{2}$ Rodrigo Parra y Leonor Zubieta, La Imagen del Maestro en la Escuela Campesina, Bogotá. Universidad Pedagógica Nacional, Centro de Investigaciones (CIUP), 1981.
} 
Los desarrollos teóricos más recientes sobre el tema ${ }^{3}$ conducen a las siguientes conceptualizaciones:

Es posible distinguir por lo menos dos instancias en el proceso de socialización. La socialización primaria, ocurrida en la infancia y entendida como la internalización —hasta hacerlo realidad subjetiva- del mundo objetivo de los significantes, generalmente los padres. Y la socialización secundaria corno cualquier proceso posterior que lleva a la internalización de submundos institucionales o basados en instituciones.

El surgimiento de la socialización secundaria supone la complejización de la organización social determinada por una particular división social del trabajo y por la distribución social del conocimiento correspondiente. Este conocimiento es de tipo especializado, por lo cual puede definirse la socialización secundaria también como “... la adquisición del conocimiento específico de roles, estando éstos directa o indirectamente arraigados en la división del trabajo"4.

Analíticamente es posible definir un continuo entre dos polos: el de la socialización completamente exitosa en el cual se ubicarían aquellos individuos que llegan a ser exactamente lo que la organización social suponía que fueran, y el del fracaso absoluto de la socialización en el otro. Sin embargo, la socialización siempre se realiza en contextos históricos y sociales muy precisos, en un mundo cuya riqueza desbordaría cualquier esquema lineal que se propusiera para el proceso de socialización y que convierte en imposible social la situación de los extremos. Por eso los resultados concretos de la socialización no solamente deben encontrarse en algún punto intermedio entre aquellos sino que remiten a la consideración de situaciones diferentes como la de una socialización ya no deficiente sino inacabada, si se tiene en cuenta que la creación social de la realidad es también un proceso continuo y contradictorio, o como la de la resocialización que cobra vigencia cuando coexisten en una sola estructura social proyectos sociales diferentes y un individuo, socializado inicialmente en cualquiera de ellos, debe por distintas razones convertirse en un miembro efectivo de cualquiera de los otros. Sea cual sea el caso de la naturaleza de la socialización, lo específico y significativo del proceso es la relación realidad subjetiva-realidad objetiva. El propósito claro de cualquier situación de socialización en cualquiera de sus momentos es, en últimas, el que el individuo haga suya, interiorice hasta identificarse con ella, la realidad que se le presenta como objetiva.

En la socialización primaria el niño internaliza el mundo de sus mayores como el único mundo posible, el único concebible, y esto no sólo porque su relación con ellos tiene inicialmente por lo menos- el carácter de inevitable, sino por la carga emocional que implica el hecho mismo de la identificación. De allí que cualquier socialización posterior comience típicamente a "adoptar una afectividad que recuerda la niñez cuando busca transformar radicalmente la realidad subjetiva del individuo" ${ }^{5}$. De hecho todo proceso de socialización intentado después de la socialización primaria, debe afrontar y tratar de resolver los problemas que originan las incoherencias existentes entre la nueva realidad y aquella ya internallizada y que tiende a persistir.

En la socialización primaria se aprehenden elementos normativos, cognoscitivos y afectivos y el vehículo socializador por excelencia es el lenguaje. Con el lenguaje y por él

\footnotetext{
${ }^{3}$ Particularmente los planteados por la escuela fenomenológica orientada por los trabajos filosóficos y sociológicos de Alfred Schutz (1899-1959).

${ }^{4}$ Peter Berger y Thomas Luckmann, La Construcción Social de la Realidad, Buenos Aires, Amorrortu editores, 1968.

${ }^{5}$ Ibidem, p. 178. 
se internalizan esquemas motivacionales e interpretativos y hasta los primeros elementos de legitimación de la realidad. La socialización secundaria implica también la adquisición de un lenguaje, del lenguaje específico de un "rol" y, con frecuencia, la construcción de un universo simbólico que a la vez que procura la coherencia entre el mundo asumido en la socialización primaria y el de esta segunda, hace posible el mantenimiento y la confirmación de la nueva realidad.

Naturalmente que toda la situación social del individuo incide en la confirmación de su realidad subjetiva, pero en la vida cotidiana es el lenguaje y sobre todo la actualización de su capacidad de aprehender y producir el mundo que es el diálogo, el instrumento fundamental del mantenimiento de la realidad. Un diálogo que para resultar eficaz a ese propósito debe ser coherente y continuo y que comprende no solamente el aparato conversacional de la comunicación oral sino también la comunicación no verbal de las expresiones y actitudes presentes en la interacción.

En alguna época se creyó que los contenidos del proceso de socialización denominado educación correspondían sin más a los conocimientos, valores, normas, etc., propios del estado del desarrollo de un país, que la escuela era la mediadora pasiva en el proceso de apropiación individual de la cultura de una sociedad. Hoy son muchos los factores que se aluden para invalidar la idea de que la función de la escuela sea simplemente transmitir la cultura de las generaciones anteriores a las nuevas. Piénsese, por ejemplo, en la inexistencia de una cultura única y completamente coherente, en las diferencias del capital cultural $^{6}$ apropiado por los distintos sectores sociales de la población desde la socialización primaria, en la dialéctica de las relaciones que se establecen entre la institución escolar y las diferentes comunidades en las que se inserta, en la presencia de otros agentes socializadores con visiones del mundo a veces discrepantes de aquella que pretende construir la escuela, en la idiosincrasia de los educadores que es el resultado de su propia historia, de su propia biografía y que los hace intérpretes activos, recreadores de la realidad cultural aprendida en la institución educativa. Por eso conocer realmente la naturaleza y el carácter del proceso de socialización realizado por cualquier sistema educativo implica, en buena medida, estudiar la institución escolar en su cotidianidad, en su y con su contexto social y cultural y desde la perspectiva de cada uno de sus actores, con la convicción de que para entender sus procesos y su realidad es necesario devolverles la voz, su lenguaje y la posibilidad de expresar la manera como ellos mismos viven sus propias circunstancias. Lo que intenta exponerse a continuación es precisamente el resultado de esa práctica en dos contextos marginales colombianos.

\section{La marginalidad}

Surgió en el país, paralelo a su proceso de industrialización-urbanización, el mito de la democratización de la educación como la condición necesaria y suficiente para obtener los niveles de participación social, económica y política propios de una organización social democrática y homogénea.

Este modelo comenzó a desvanecerse, como lo han indicado diversos estudios ${ }^{7}$, y la efectiva expansión del sistema educativo ${ }^{8}$ no condujo ni a más equitativa distribución del

\footnotetext{
${ }^{6}$ Pierre Bourdie y Jean Claude Passeron, La Reproducción, Elementos para una Teoría del Sistema de Enseñanza, Barcelona, Ed. Laia, 1977.

7 Jean Pierre Jallade, El Gasto Publico para la educación y Distribución del Ingreso en Colombia, World Bank Staff Papera No. 18, The John Hoopkins Presa, 1974. Guillermo Briones, "Educación y Estructura Social", Recia fa Colombiana de Educación, Universidad Pedagógica Nacional, Centro de Investigaciones, Bogotá, No. 1, 1978.
} 
ingreso, ni a una mayor movilidad social, ni a la configuración de un mercado laboral cuya dinámica permitiera la vinculación productiva a mayores sectores de la población potencialmente activa.

En cambio surgió el proceso de devaluación de la educación ocasionado por la disparidad entre los ritmos de crecimiento del sistema educativo y la estructura ocupacional, y cuyo rasgo mas notable consiste en los cada vez mayores requisitos educativos exigidos en el mercado de trabajo para acceder a cualquier nivel de su estructura.

En este panorama, las consideraciones sobre la relación entre marginalidad y educación también han tenido adecuaciones, variaciones y reinterpretaciones. En sus comienzos participaron de la euforia colectiva y creyeron ver igualmente en el acceso a la segunda la posibilidad de salir de la primera. En efecto, si la situación de marginalidad era entonces un problema de "actitudes" y de "motivaciones", la educación se vislumbraba como la solución por su presunta incidencia en el cambio de aquellas.

Los desarrollos siguientes de la teoría marginalista o, mejor, de sus críticas, propusieron una interpretación de la marginalidad hoy comúnmente aceptada, como aquella situación de exclusión que experimentan algunos sectores de la estructura social que se rigen por relaciones de producción diferentes a las del modo de producción dominante pero que cumple para este funciones políticas y económicas definidas. Esta perspectiva diferente cuestiona la visión dualista de la sociedad implícita en la concepción marginalista original y pone en entredicho la función integradora de la educación como solución al problema de la marginalidad, pero no invalida el uso del concepto para referirse a las condiciones sociales que requirieron su aparición: La existencia de comunidades con una población vinculada de manera indirecta y aparentemente difusa a los procesos productivos, a los de participación cultural y política y excluida a la vez, en mayor o menor medida, de los de distribución de los bienes y servicios generados socialmente.

El análisis social se mueve hoy dentro de los parámetros teóricos del desarrollo desigual en los que la situación de marginalidad se concibe también como condición estructural de un sistema que la precisa para su propio sostenimiento y reproducción.

\section{Los contextos sociales}

Los estudios sobre educación y marginalidad parten de una hipótesis sobre la naturaleza desigual del desarrollo colombiano que plantea la coexistencia de diferentes formas productivas. Una exposición detallada de la misma sobrepasa las intenciones de este artículo. Por esa razón sólo se describirá aquí, de manera general, la conformación de la sociedad colombiana.

Como resultado de los procesos de urbanización e industrialización iniciados en Colombia en la posguerra surgió con mayor énfasis la diferenciación entre las zonas rural y urbana. Todos los indicadores socioeconómicos coinciden en señalar al sector rural como un sector deprivado en relación con las condiciones de vida características del urbano.

\footnotetext{
${ }^{8}$ Para el período 1964-1977, por ejemplo, el crecimiento de la matrícula fue del $92 \%$ en la primaria, 314\% en la secundaria y $527 \%$ en la superior (Información del Departamento Nacional de Planeación). 
Al interior de cada una de las zonas, a su vez, existen diferencias regionales que hacen posible hablar tanto de contextos rurales como urbanos marginales e integrados. En términos generales se identifican como contextos urbanos y rurales integrados aquellos sectores caracterizados por una organización social y unas formas productivas definidas por las leyes del mercado.

La marginalidad tanto rural como urbana requiere algunas precisiones que se harán posteriormente.

\section{Educación y marginalidad rural}

\section{A. Los rasgos de marginalidad de las comunidades Campesinas}

Aunque no constituyen su configuración más típica, las comunidades campesinas son expresión de la marginalidad en el medio colombiano. La economía, denominada campesina por ser exclusiva de estos contextos, se dedica prioritariamente al autoconsumo y en menor medida produce alimentos para los centros urbanos.

Esta es precisamente su contribución al sostenimiento de la estructura social, aunque sus formas de producción basadas en la pequeña propiedad y en el empleo casi exclusivo de la fuerza de trabajo familiar la hagan parecer desarticulada en un sistema económico organizado según las leyes capitalistas del mercado.

Con todo, el rasgo más notable de la condición marginal de las comunidades campesinas, y el más específicamente relacionado con el proceso educativo, lo constituye su anacronismo cultural. No se cuenta todavía con estudios suficientes para hacer un análisis de la cultura campesina, pero es posible hacer ya algunas consideraciones.

El mundo cultural campesino se circunscribe con frecuencia a los propios límites geográficos que encierran una comunidad. Su contacto con la realidad exterior, con la sociedad más amplia, es esporádico, irregular o de poca significación en términos de las motivaciones y de los problemas que reclaman la atención del campesino cotidianamente. Por tradición se establecen el tipo y los modos de trabajo, las pautas de comportamiento social, las líneas, las manifestaciones y los depositarios de la autoridad y hasta una visión del mundo y de la vida muy rica en interpretaciones pragmáticas, mágicas y místicas pero extrañas a los procesos mentales de abstracción que exige para el hombre común de contextos más urbanos el conocimiento, así sea funcional, de su realidad.

De allí que lo inmediato en el plano vivencial y lo útil a nivel valorativo constituyan elementos fundamentales de la cultura campesina que permean toda su existencia, incluso las expresiones artísticas en las que, en muchos casos no caben manifestaciones que, como el teatro, implican la capacidad de concebir como realidad lo imaginado. A cambio, la simbiosis particular del hombre campesino con su medio condiciona y a la vez requiere no sólo el desarrollo de una agudeza sensorial especial sino de un sentido de lo práctico en general ajeno al hombre citadino. La organización de la vida y de las mismas relaciones se rige por las exigencias que el medio natural le hace al hombre antes que por las que pueden provenir de su medio social. Y éste a menudo se limita al círculo familiar y al de algunos pocos vecinos o habitantes del poblado cercano con los que establece relaciones que, como las de compadrazgo, giran siempre en tomo a la contraprestación de servicios. 


\section{B. Funciones de la escuela en el contexto campesino}

En general, puede pensarse que la escuela lleva a cabo cuatro funciones fundamentales en la sociedad colombiana: 1) una función de enseñanza propiamente dicha y cuyos problemas se plantean tradicionalmente dentro del plano cognoscitivo. 2) Una función que hace relación a la producción, a la formación de la mano de obra, y que se aplica con mayor claridad en los contextos urbanos. 3) La función de transmitir de manera activa los valores sociales. 4) Y una cuarta función, notable en los contextos campesinos, como es la de ser una institución integradora de los individuos en valores y conceptos que provienen de la cultura urbana y que tienen que ver tanto con la formación de conceptos como los de región, de nación, de pensamiento científico como con la visión del mundo de clase media urbana que trasmite el maestro. En efecto, si se tiene en cuenta la organización relativamente elemental de la vida social campesina, en la cual la poco compleja visión social del trabajo y la distribución social del conocimiento se resuelven mediante la asignación de roles por edad y sexo, una socialización secundaria, posterior a la primaria realizada por el grupo familiar, parece superflua. Así por lo menos deben percibir algunos padres de familia que niegan a sus hijos la posibilidad de asistir a la escuela con el argumento de que ellos nunca lo hicieron y sin embargo nunca les resultó necesario ni para trabajar la tierra ni para sostener a la familia.

\section{Rol docente e imagen del maestro}

Desde esta perspectiva, entendiendo la escuela como mediadora o vínculo entre distintos contextos socioculturales, el rol docente adquiere dimensiones más amplias que las comúnmente enunciadas en los trabajos existentes sobre el maestro rural. Tradicionalmente, cuando se hablaba del maestro de comunidades campesinas, se le señalaba como líder o promotor de cambio y se asumía que debía interesarse por todas y cada una de las actividades que se adelantaran por el progreso de la vereda o que debía ser él quien las impulsara. Esta concepción partía del supuesto de que la diferencia de nivel educativo y la oportunidad de interacción brindada por la escuela eran factores suficientes para que el maestro asumiera una posición de liderazgo. Todavía los últimos planes y programas de desarrollo ${ }^{9}$ insisten en ubicar tanto al maestro como a la escuela veredal en ese rol, pero otra es la situación creada por la pérdida de status de la profesión, la generalización de una concepción menos "apostólica" y más contractual de la labor docente, el incremento de la conciencia reivindicativa y gremial del magisterio, el fracaso mismo de los programas de desarrollo rural que han socavado la credibilidad tanto en ellos como en sus impulsores, la implementación de distintas condiciones laborales como la jornada única, la posibilidad de vivir fuera de la escuela y su comunidad o la poca estabilidad y escasa permanencia del maestro en una misma escuela. Por eso un estudio reciente ${ }^{10}$ pudo llegar a la conclusión de que el ser líder o promotor de cambio social dista mucho de ser hoy, como sí debió suceder hace algunos años, un aspecto imprescindible de la caracterización del maestro rural y de las expectativas de logro de todo docente que quiera ejercer en el área.

Transmitir conocimientos era y es otra de las funciones que debe desempeñar el maestro en todos los contextos y que define otra de las dimensiones de su rol. En el medio campesino esta labor es particularmente compleja y difícil por diversas razones. Algunas se mencionan en los diagnósticos oficiales como los factores que explicarían el escaso rendimiento académico y la poca retención observada en la escuela rural:

\footnotetext{
${ }^{9}$ Plan Nacional de Alimentación y Nutrición (PAN), Programa de Desarrollo Rural Integrado (DRI).

${ }^{10}$ Dieter Pasa, El Maestro Rural Fund. Friedrich Naumann, Bogotá, 1979.
} 
desnutrición, participación temprana del niño en tareas agrícolas y domésticas, escasez de recursos, instalaciones inadecuadas, número insuficiente de aulas y de docentes y, sobre todo, la insuficiente capacitación de los maestros.

El Capital Cultural de las comunidades tiene también mucho que ver en las dificultades que encuentra el maestro para realizar la instrucción en el campo. La inexistencia del aprestamiento, la escasa o ninguna familiaridad del niño con algunos de los temas tratados en la escuela, el empleo de un lenguaje desconocido, la exigencia de procesos de razonamiento contrarios o, por lo menos distintos, a aquellos a los cuales el niño está acostumbrado, son, entre otros, algunos de los problemas con los que tropieza el docente en su condición de comunicador del saber social. A ellos parecen referirse las críticas formuladas desde distintos sectores cuando señalan la poca flexibilidad del currículo o su inadecuación a las condiciones regionales.

Existe además otra dimensión del rol docente que, de acuerdo con la discusión anterior sobre las funciones de la escuela, adquiere en el contexto campesino una significación especial: su condición de intérprete entre dos mundos culturales diferentes. Sin duda que el transferir conocimientos es un elemento constitutivo de esta dimensión pero no la agota. Diríase más bien que el maestro en su condición de mediador realiza las tareas de instrucción, pero también las de inculcación de hábitos, actitudes, motivaciones, concepciones, valores, modelos, aspiraciones, conductas y comportamientos. En realidad unas y otras son las propias de toda educación que se conciba como instrucción y como formación. Lo que resulta diferente en la situación del contexto campesino es el contenido específico de las mismas, su relación con el proceso de socialización primaria del niño en la familia, los factores que en este medio pueden hacer o hacen eficiente la labor del maestro.

Es decir, la naturaleza, el contenido, los medios y las consecuencias del proceso de socialización secundaria efectuado por la escuela.

\section{La socialización}

Como ya se anotaba, la organización social típica de las organizaciones campesinas es relativamente sencilla. Cada individuo asume diferentes tareas y desarrolla distintas actividades según sea su sexo y su edad. En épocas normales los hombres cultivan la tierra y las mujeres se dedican a las labores domésticas y a la crianza de los hijos. En los días en los que deben intensificarse las labores agrícolas las mujeres incrementan su participación en ellas e igual hacen los hijos e hijas menores. Los conocimientos y habilidades necesarios en una y otra situación se aprenden de los miembros mayores de la familia y en la experiencia diaria. Adecuar los contenidos de la educación a estas condiciones significaría tal vez suplir a la familia en tales funciones, pero eso no es lo que hace allí la escuela.

En realidad, tanto desde el punto de vista de la instrucción corno desde el de la formación, la escuela rural sólo es rural por su ubicación física. Sus contenidos, su método y la formación del maestro, visto aquí como el agente socializador, son urbanos ${ }^{11}$. Proceden de una realidad económica, social y cultural diferente, de una realidad objetiva distinta, y la eficacia de su acción implica que el niño campesino, socializado inicialmente

\footnotetext{
${ }^{11}$ Ver al respecto: Rodrigo Parra, María Elvira Carvajal y Carlos Francisco Parra, La Profesión de Maestro y el Desarrollo Nacional, Bogotá, CIUP.
} 
en el mundo propio de su familia, internalice hasta identificarse y ubicarse en ella, esta segunda realidad que le trae la escuela.

A diferencia de lo que sucede en el contexto cultural y social original de la institución educativa, la educación primaria no es aquí una etapa indispensable de la secuencia de formación y aprendizaje socialmente requerida para llegar hasta el eficiente desempeño de uno cualquiera de distintos roles, sino el proceso mediante el cual ha de transformarse esa primera realidad subjetiva asumida por el niño. La labor real de la educación primaria, tal como actualmente existe en las comunidades campesinas, se acerca mucho más, cuando tiene éxito, a una acción de resocialización que a una socialización secundaria. Es en ese sentido que debe entenderse su función integradora.

Plantear así las cosas implica mirar el fenómeno educativo desde la perspectiva de la comunidad campesina. Es desde su realidad que la acción de la escuela se revela corno la inducción hacia una existencia social y cultural ajena. Pero aunque aislado y marginal, el contexto campesino no es ni estático ni autárquico. Los análisis de las tendencias del desarrollo nacional, por el contrario, le atribuyen características de franca desintegración: altas tasas de migración, ingresos familiares provenientes de la combinación del trabajo en el predio con trabajo asalariado, disminución paulatina pero constante del tamaño de las propiedades, competencia de las empresas agrícolas en el mercado, márgenes escasos o negativos de rentabilidad, etc. Desde esta otra perspectiva es un hecho que la escuela introduce a las generaciones jóvenes de los contextos campesinos en una realidad que aunque todavía no sea la suya sí van a tener que conocer. Por ello la naturaleza tan compleja y contradictoria de los resultados de la resocialización. Entre más exitosa sea la escuela, las relaciones del individuo con su medio original tenderán a hacerse cada vez más conflictivas (problemas de interacción, de identidad, de ubicación, etc.), pero a la vez resultará más apto para moverse en el mundo del que proviene la escuela. Esta es la paradoja que debe enfrentar la planeación educativa y en primera instancia el maestro rural. Qué tan capacitado esté para hacerlo con habilidad es difícil decirlo en razón a que los estudios de este tipo apenas se inician en el país, pero la conclusión aportada por la investigación de la escuela en la marginalidad urbana no permite un pronóstico muy favorable: “... parecería evidente que los puestos de trabajo en áreas marginales actúan como puestos de entrada a la carrera docente. Asimismo, la flauta (implícitas la mayor parte de los casos) de distribución de cargos indican que la entrada se efectúa por los grados claves (el primero, fundamentalmente). Esta modalidad muestra un hecho muy particular con respecto al funcionamiento del mercado de trabajo en relación con la calidad del producto que se desea obtener. Parecería, en efecto, que el mercado de trabajo de los educadores funciona --en los contextos marginales-- con una dinámica que no tiene en cuenta la calidad del producto. Haciendo una analogía con los sectores productivos, podría sostenerse que en ningún proceso de este tipo se entregan las etapas más delicadas de la producción a los aprendices o al personal con menos experiencia y menos calificación"12

\section{E. La Resocialización y la relación maestro-alumno ${ }^{13}$}

Como todo intento de resocialización, el de la escuela requiere también y ante todo de la creación de condiciones similares a las que rodean a la socialización primaria para que

\footnotetext{
12 Juan Carlos Tedesco y Rodrigo Parra, op. cit., págs. 14-15.

${ }^{13}$ Los textos que aquí aparecen a manera de ilustración corresponden a extractos de entrevistas realizadas durante el trabajo de campo de la investigación sobre la imagen del maestro rural ya citada. Felipa es la maestra de una de las veredas en las cuajes se desarrolló el trabajo.
} 
la realidad subjetiva del niño pueda efectivamente transformarse. El maestro no tiene control sobre la situación social total del alumno pero sí puede tenerlo de la vida cotidiana en la escuela, en el aula y del tipo y calidad de la relación que establezca con los estudiantes y la comunidad. Que el maestro pueda reproducir aproximadamente las condiciones que rodearon a sus alumnos en la socialización primaria significa que conoce la comunidad en la cual trabaja, que es capaz de identificarse y de comprometerse en alguna medida con ella, que puede lograr una relación maestro-alumno con una fuerte carga emociona positiva y que logra ser aceptado y respetado en la comunidad. Estas son las manifestaciones más significativas de lo que se podría llamar la afectividad en las relaciones. En otras palabras, la naturaleza afectiva de las relaciones es una condición indispensable para el éxito de la resocialización.

\section{FELIPA:}

"Y así me han sucedido casos!!... Digo que tengo que expulgarlos, que sacarles la cantidad de piojos, que bañarlos, de todo... entonces ellos ven en mí más bien una mamá..., ellos en mí tienen una confianza muy grande y prácticamente no sólo en esta escuela sino en todas. Yo comencé así.., y ya después de tenerse uno al muchachito ganado, todo es tan fácil! $Y$ por medio de ellos uno se va haciendo también a los padres de familia".

\section{ALUMNO DE FELIPA: (Texto escrito)}

Pregunta; ¿Qué piensa de su maestra?

---Que es muy buena que tiene paciencia para esplicarnos que nos confia cualquier cosas que tiene libro para esplicar bien las cosas que nos quiere y por eso la queremos que ella no nos pega que nos enseña a leer a escrivir a sumar.

(Redacción y ortografía idéntica al original)

\section{PADRE DE FAMILIA:}

"Todos aquí vivimos agradecidos con Doña Felipa por sobre todo lo bien que sabe tratar a los niños y a la gente, por la paciencia y decencia que tiene con todos... siempre nos ha ayudado cuando hemos ido a pedir alguna cosa

Felipa encarna, sin que haya mediado un particular proceso reflexivo sobre la naturaleza de su labor, una concepción tradicional del magisterio. La institucionalización de la profesión docente es la consecuencia del cada vez más amplio radio de influencia de las relaciones salariales concomitantes a la industrialización y a la urbanización. Las relaciones maestro-alumno, por lo tanto, también han cambiado. Se trata ahora de relaciones impersonales cuya interacción se limita a la establecida en las aulas de clase mediatizadas por un consistente y preestablecido conjunto de conocimientos que, se supone, debe transmitir el primero a los segundos. El maestro tradicional por el contrario, estaría en condiciones más ventajosas para lograr el clima afectivo apropiado para la resocialización. Este es quizá uno de los rasgos rescatables o por lo menos deseables de la concepción tradicional del magisterio.

Por otra parte, la confirmación y el mantenimiento de la realidad aprehendida por el niño en la escuela exigen la continuidad y la coherencia del diálogo. No basta para ello, aunque sí es imprescindible, que el maestro fomente y estimule la espontaneidad expresiva del niño, que posea un manejo hábil del lenguaje, que procure conocer las 
características y las particularidades del que se habla en la comunidad, que no pierda credibilidad ante sus alumnos actuando en contradicción con lo que dice. Estos son aspectos más o menos manejables por parte del maestro. Pero las características mismas de los consumidores campesinas conllevan factores de inconsistencia, de irregularidad y de contradicción en unos casos imposible y en otros difícil de ser modificados por el docente y que son fuente permanente de conflicto.

\section{MADRE DE FAMILIA:}

Doña Felipa es muy buena maestra... Ella ha hecho por la verdad lo que ha podido y eso que a veces la gente no le colabora y sola no puede hacer más. Eso ha tenido disgustos hasta en cosas de los niños de la escuela... Una cosa es que ella les hable de educación en palabras y les enseñe a leer y a escribir y todo lo que tiene que enseñarles, pero es en la casa con el ejemplo y corrigiéndolos donde los niños tienen que aprender a ser decentes y correctos, a no hablar mal, a respetar lo ajeno... qué saca la maestra siendo tan decente con los niños si no hay que ver lo que esos niños oyen en sus casas!

Para garantizar en alguna forma la continuidad del diálogo, en la escuela entra a ser de primordial importancia la duración de la escolaridad, la existencia de oferta escolar de mayor o menor duración y las situaciones culturales o económicas que impiden o traban la asistencia del niño a la escuela. Este fenómeno ha sido tratado generalmente a nivel de costos de la educación y se ha demostrado que los costos causados por ausencias de maestros y alumnos asumen características muy graves. Consecuentemente también es importante su papel en la internalización de la realidad que se propone generar la escuela. Esta es otra manera de considerar los efectos negativos de la deserción escolar. Una deserción que no siempre está fuera del control del propio sistema educativo como la generada por las actitudes del maestro y por las características de su práctica pedagógica.

\section{FELIPA:}

$\mathrm{Si}$, eso a uno se le presentan muchas cosas en la escuela y, a veces, cuando uno no sabe entrevistarse con las personas, surgen peores cosas, cuando la maestra también se levanta, se alza. Que usted no me viene aquí a poner órdenes, yo mando y punto. Y si no le gustó saque a su niño o lo suspendo.

Precisamente en la reunión del lunes el señor jefe de grupo nos habló de eso. De la cantidad de niños que las maestras están suspendiendo sin escrúpulos. Los mandan para la casa, y eso es marginar al muchacho! El muchacho ya se manda para la casa cuando verdaderamente sea una expulsión! Cuando es un caso grave! Pero hay otras cosas que se pueden superar. Entrar con una matrícula de cuarenta y cinco y yo me aterro de que termine dizque con dieciséis!

\section{F. La socialización en contextos marginales urbanos e integrados}

La naturaleza de la socialización que realiza la escuela en los diferentes contextos sociales no es igual, como no son iguales las funciones sociales que aquella cumple en cada uno de ellos.

Las condiciones de marginalidad tampoco son idénticas en todos los sectores. Igual que la rural, la marginalidad urbana puede definirse por sus rasgos de exclusión; pero la 
exclusión aquí adopta otras dimensiones que la hacen específica y que en buena medida condicionan la acción de la institución educativa.

Siguiendo el análisis presentado en el estudio "Marginalidad Urbana y Educación Formal" ya citado, pueden sintetizarse así las características de la primera:

En primer lugar, la percepción social la identifica con la localización geográfica. Independientemente de que al interior de los "barrios marginales" sea posible diferenciar, como en efecto se encontró en el estudio, entre sectores de población integrados y excluidos en términos tanto de su situación socioeconómica como cultural, desde el conocimiento de sentido común del ciudadano corriente esta heterogeneidad no existe.

En segundo lugar, la desvinculación económica de estos sectores —inestabilidad e improductividad laboral, desempleo, insuficiencia de los ingresos - no inválida la concepción estructural de la marginalidad. Ya se ha demostrado suficientemente para toda América Latina que el subempleo se traduce en ocupaciones que como las calificadas como "servicios personales" garantizan un determinado nivel de vida en las ciudades, y el desempleo en una oferta excesiva en el mercado de trabajo que incide en el sostenimiento de determinados y bajos niveles salariales.

La exclusión cultural también tiene características propias y fundamentales para entender la acción de la escuela en los contextos marginales urbanos. Se generarán allí ciertas estructuras de supervivencia de las que resultan esencialmente dos tipos de relaciones sociales: el intercambio entre iguales (intra e inter familias) y las relaciones asimétricas que permiten la articulación entre los marginales y el sector formal a través de intermediarios que aunque emergen del sector marginado, poseen algún tipo de calificación o recurso que les permite la vinculación.

Por otra parte, mientras las relaciones asimétricas son inestables, las de reciprocidad entre iguales son las que ofrecen un nivel mínimo de seguridad a sus participantes y garantizan, relativamente, la subsistencia material.

Pero quizá el aspecto más importante para tratar de definir el mundo cultural del marginado sea el de la existencia, al interior de las relaciones de reciprocidad, tanto de estructuras muy fuertes de solidaridad hacia adentro con otras igualmente sólidas de agresión hacia afuera. Mientras las relaciones entre iguales se rigen por códigos morales de reciprocidad, las de los marginales con los sectores integrados de la sociedad global lo hacen generalmente por otros de antagonismo.

Este doble carácter del ámbito cultural plantea a la acción socializadora de la institución educativa una ambigüedad que con frecuencia ella misma reafirma en su práctica cotidiana. Aunque la escuela intenta neutralizar las formas estables de solidaridad promoviendo un tipo de comportamiento fundamentalmente individualista y competitivo (propio de las relaciones de mercado predominantes) y sancionando cualquier expresión de agresividad y violencia entre los alumnos, “... las observaciones indican que si bien la escuela parece haber logrado neutralizar la aparición visible de las asociaciones grupales en su interior, no controla las relaciones de agresión que se establecen entre los mayores y los menores en los momentos en que éstos interactúan dentro de la escuela. Esta interacción se da fundamentalmente en los recreos y allí la acción de los docentes es prácticamente nula... Además, estas expresiones son visibles en la acción externa hacia la escuela, ya que las agresiones que provienen del exterior (y que son frecuentes) 
parecen ser producto de grupos de adolescentes que mantienen o mantuvieron recientemente vínculos con la escuela en calidad de alumnos"14.

Tales hechos llevan a pensar que la escuela difícilmente logra modificar la realidad subjetiva inicialmente interiorizada por los alumnos en su medio cultural de origen y que la aparente aceptación de las imposiciones escolares por alumnos y padres de familia sólo tienen un carácter manipulativo. Unos y otros internalizan la realidad que les presenta la escuela pero no para hacerla suya sino para utilizarla con propósitos precisos.

Los alumnos, por ejemplo, para evitar las sanciones, los castigos. Los padres de familia para asegurarse de que sus hijos sean recibidos en la escuela, no porque importe mucho lo que en ella puedan aprender, sino porque resulta el mejor sitio para dejar a los hijos mientras trabajan.

No cabria esperar aquí que la afectividad como ya fue definida y como forma de la relación maestro-alumno tenga la misma efectividad que en los contextos campesinos. El maestro encuentra demasiados obstáculos para crear un clima afectivo y condiciones similares a las vividas por el niño en la socialización primaria. Piénsese por ejemplo en hechos como los siguientes:

La socialización primaria del niño es con frecuencia un proceso complejo por la presencia de varios y contradictorios socializantes o agentes socializadores.

-El carácter antagónico que tienen las relaciones entre la cultura propia de la escuela y la de la comunidad en la cual se inserta.

Las mismas características del docente que según los resultados del estudio sobre la profesión maestro ${ }^{15}$ posiblemente sea muy joven, inexperto, haya migrado a su vez de contextos urbanos menos industrializados o del rural y asuma su labor en una perspectiva de transitoriedad, ya hacia sectores integrados, ya hacia otra profesión si es que estudia simultáneamente otra carrera.

- Las condiciones laborales que hacen del magisterio de los sectores marginados un sector a su vez marginado en el contexto del sistema educativo.

Poco a poco la permanencia en los sectores marginales exige de la escuela y sus docentes procesos adaptativos, que si bien garantizan una mayor aceptación de la institución educativa en el medio, neutralizan o disminuyen considerablemente el efecto de transformación cultural de aquella en éste. Esto es lo que sucede cuando los docentes más jóvenes adoptan el estilo de trabajo tradicional de los maestros mayores ante su propia "Anomia" metodológica, cuando se enfatizan en la escuela las prácticas autoritarias con el argumento de que sólo con ellas se puede lograr cierto orden puesto que es la forma de autoridad a la que están acostumbrados los niños, cuando se concentra la autoridad en una figura masculina así ella no esté a su cargo dentro de la estructura organizativa de la escuela o cuando se pretende defender la estabilidad de la organización social lograda en la institución aislándola de toda influencia externa y siguiendo los mismos patrones de las relaciones de reciprocidad existentes entre los marginados. En síntesis, podría decirse que la escuela misma acentúa la disociación cul-

\footnotetext{
14 Juan Carlos Tedesco y Rodrigo Parra, Op. cit.. pág. 6.

${ }^{15}$ Rodrigo Parra, María Elvira Carvajal. C. Francisco Parra, Op. cit. 
tural de los alumnos, o mejor dicho, que “... concreta la disociación cultural que existe a nivel de la estructura social en el plano de los hábitos internalizados por cada individuo" 16

En los contextos integrados la situación es muy distinta. La tarea de socialización de la escuela sí puede interpretarse en ellos como una socialización secundaria estrictamente hablando, en la medida en que la formación escolar es parte de la secuencia socialmente definida como la vía normal de acceso a cualquier rol dentro de la estructura, y a que la cultura de la escuela guarde relaciones de reciprocidad, legitimación y coherencia con la propia de la socialización primaria. Naturalmente que no se trata aquí tampoco de un proceso lineal y libre de conflicto, pero los problemas son de otra índole. En efecto, si se tiene en cuenta el origen social de clase media del docente como tendencia general, a medida que se asciende en la escala social va resultando que el capital cultural apropiado por los alumnos en la socialización primaria, en sus hogares, es más amplio que el propio del docente (Bilingüismo, mayor familiaridad con valores estéticos y con las concepciones políticas y económicas que rigen el país, etc...). En esas condiciones el desarrollo de la afectividad en la relación maestro-alumno no sólo es innecesaria sino difícil, y en cambio se plantean conflictos relacionados con la legitimación y el ejercicio de la autoridad del maestro. Casi podría decirse que en estos contextos es el maestro quien resulta marginado y que en ningún contexto como en éste la escuela cumple mejor su función ideológica de legitimación de la estructura clasista de la sociedad. 